\title{
Assessment of knowledge and Practicing Regarding Breast Self - Examination Among Female Students At South Valley University
}

\section{Sayed Ahmed Taha MD}

Departments of Obstetrics \& Gynecology,

Faculty of Medicine, South Valley University, Kena

\section{Manal Farouk Moustafa MD}

Department of Obstetrics \& Gynecology,

Faculty of Nursing, Assuit University South Valley Universities (Kena), Egypt

\section{Sahar Fahmy Gawad MD}

Lecturer of Obstetrics \& Gynecology, Faculty of Nursing,

South Valley Universities (Kena)

\section{Reda Refahi Ali Ms Sc}

Department of Obstetrics \& Gynecology,

Faculty of Nursing, South Valley Universities (Kena), Egypt

\begin{abstract}
:
Objectives: To assess the knolwedge of univeristy female students about breast self examination (BSE) including the facilities, sources and the impact of residence and level of parents' education on these knolwedge.
\end{abstract}

Subjects and Method: The study included all female students of faculties of Specific Education (Group I) and Archaeology (Group II), South Valley University, Qena, Egypt. The study relied on a specially designed self-administered questionnaire developed by the researchers and consisted of three parts: The first part included collection of personal data, the second part was concerned by the knowledge of students about BSE, and the third part was concerned by the performance of BSE.

Results: Radio and TV constitute the main source for $36.3 \%$ of females had knowledge, reading about $\mathrm{BSE}$ and communication facilities on the internet were the following sources in Group I, while personal communication data sources are the main in group II with significant difference of distribution according to source of knowledge between both groups. Only 63 females do BSE, 30 do it monthly, 45 do it 6 monthly and 25 do it 
yearly; 18 females do it before date of menstrual period, 21 do it after period and 24 females do it at any time with non-significant difference between both groups. There was non-significant difference between both groups as regards the method of performing the BSE and the feedback action to notice breast changes. For both groups, irrespective of rural or urban inhabitance, the majority of females had their information through radio and TV, books and magazines are highly used by rural females of group I but by urban females of group II. Rural inhabitants performing BSE was significantly higher in both groups compared to urban inhabitants. There was significant difference between both groups categorized according to residence as regards knowledge about the methods of BSE. Educated mothers significantly affected their girls knowledge and application of facilities for gaining information and significantly higher frequency of proper choice of timing of BSE.

Conclusion: Knowledge about BSE is still underdeveloped and controlled by the residence, level of education of the mothers, the availability of knowledge providing facilities and even these knowledge needed to be corrected as regards how and when to practice BSE. Thus mass surveys for provision of knowledge among higher school and university student is mandatory to develop and correct their knowledge about BSE

Keywords: Cancer breast, Breast self-examination, Screening, Questionaire

\section{Introduction}

Cancer is becoming an increasingly important factor in the global burden of disease. Worldwide, the estimated number of new cases each year is expected to rise from 10 million in 2000 to 15 million by the year 2020 and out of all these new cases $60 \%$ will develop in the less developed parts of the world. Approximately 20 million people are alive with cancer at 2003 and by 2020 there will probably be more than 30 million ${ }^{(1)}$.
Breast cancer is a major public health problem for women throughout the world. In the United States breast cancer remains the most frequent cancer in women and the second most frequent cause of death. In 2007 it is estimated that breast cancer will account for $26 \%$ of cancer cases and $15 \%$ of cancer deaths, which translates to 176,296 new cases and 40, 515 deaths ${ }^{(2)}$.

According to institutional data from South Egypt Cancer Institute, breast 
cancer is the second common cancer in frequency after bladder cancer. Incidence of new cases was $11.62 \%$ relates to all new cases attended during 2005. So it is the most prevalent cancer among Egyptian women and constitutes $29 \%$ of National Cancer Institute cases. Median age at diagnosis is one decade younger than in countries of Europe and North America and most patients are premenopausal ${ }^{(3)}$.

Breast self Examination (BSE) has been advocated for years as good method for early detection of breast masses and a large percentage of women discover their breast cancer accidentally or during BSE. Women at high risk for breast cancer are especially encouraged to be attentive to the importance of early detection through routine BSE. Moreover, BSE may be of a real value for younger women who do not yet get regular mammograms ${ }^{(4,5)}$.

The effectiveness of BSE is determined by the woman's ability to perform the procedure correctly, by her knowledge of her own breast tissue, and by the density of her breast tissue. BSE: Is a method of finding abnormalities of the breast, for early detection of breast cancer. The method involves the women herself looking at and feeling each breast for possible lumps, or swelling ${ }^{(6)}$. The value of performing BSE on a monthly basis and reporting findings to the clinician should be emphasized, specially including that the treatment of disease detected early in its course is much more likely to be successful compared with treatment started later, after disease had time to progress, it should be explained that a women will become expert in knowing her own breast and when something has changed, it is then the task of clinician to determine the significance of the change. Because breast cancer considered the leading type of cancer in women, So Clinical breast examination and BSE are the secondary preventive methods used for screening in the early detection of the breast cancer ${ }^{(7,8)}$.

The current prospective study aimed to assess the knolwedge of univeristy female students about breast self examination including the facilities, sources and the impact of residence and level of parents' education on these knolwedge.

\section{Subjects and Method}

The current prospective comparative study aimed to include all female 
students of faculties of Specific Education (Group I) and Archaeology (Group II), South Valley University, Qena, Egypt. Data collection was started at Feb till May 2010 through two weekly visits to the colleage. The study relied on a specially designed selfadministered questionnaire developed by the researchers after review of literature and interviewing experts in Nursing Obstetrics and Gynecological Specialties.

The designed questionnaire consisted of three parts: The first part included collection of personal data including Student's name, age, name of faculty, residence, educational level of mother and father, occupation of mother, and facilities as computer and internet. The second part was concerned by the knowledge of students about breast selfexamination (BSE) including having knowledge about BSE or not, and the source of knowledge either mother, books, magazine, radio \& TV, friends, family, computers and internet and others as conferences and lectures. The third part was concerned by the performance of BSE including the following BSE data:
- Doing of BSE: Yes or No.

- Frequency of doing BSE: monthly, 6-monthly or yearly.

- Date of doing BSE: before or after menstruation, or no definite time.

- Methods of BSE: observation and view for the external shape, size of breast, color of breast, presence of abnormalities in breast, or all the previous. By pressure and touch using three middle fingers, by circular motion, touch all parts of breast, examination of axillary lymph nodes. By notes: record notes, detect the next exam, all the previous.

- The position preferred during examination: while standing in front of a mirror, while lying down, while taking a shower or all the previous.

- The mode of BSE: circular motion, from enter to outer, with clockwise, from upper to lower, from side to side, others.

- What parts of hands used when do exam: the palm of hands, fingertips or all hand.

- What is the feedback of finding any abnormality in breast: tell 
- mother, go to doctor, search in books and internet, go to friends or others.

- The exact time for BSE: Monthly, weekly, 7-10 days from the 1st day of menstruation, the same day from every month if not menstruation, and others.

\section{Statistical analysis}

Data collected were analyzed and tabulated and statistically compared using SPSS (Version 15, 2010) program. Data are presented and mean $\pm S D$, number, percentage and were analyzed using Chi-square to determine significance between numerical variables. $\quad \mathrm{P}<0.05$ was considered significant

\section{Results}

Table I shows that mean age of studied subjects was $19.52 \pm 2.41$ years in Group I and $19.75 \pm 2.74$ years in Group II with $77.5 \%$ and $67.3 \%$ of both groups, respectively, were in range of 17-20 year and $22.5 \%$ and $32.7 \%$, respectively were older than 20 years. About $70 \%$ and $62 \%$ of mothers of studied subjects were educated and about $83 \%$ and $62 \%$ in both groups, respectively, were educated. Majority of studied subjects; $84 \%$ in group I and $51.7 \%$ in group II were rural areas inhabitants. As regards mother job, $84 \%$ and $77.7 \%$, in both groups, respectively were housewives and remaining mothers were employee Table 2 show that hree hundreds and sixty-five subjects $(73 \%) ; 65 \%$ of Group I and $78.3 \%$ of Group II, were found to have no knowledge about BSE with significant difference in favor of Group II. As regards the source of knowledge, Radio and TV constitute the main source for $36.3 \%$ of females had knowledge, reading about BSE and communication facilities on the internet were the following sources in Group I, while personal communication data sources are the main in group II with significant difference of distribution according to source of knowledge between both groups (Fig. 1). Only 63 females do BSE, 30 do it monthly, 45 do it 6 monthly and 25 do it yearly; 18 females do it before date of menstrual period, 21 do it after period and 24 females do it at any time with nonsignificant difference between both groups,

As regards Table 3 All subjects concerned with BSE conduct examination by various methods; however, the number of females 
concerned with the presence of mass and examining their axillae for lymph nodes was higher among group I females, despite the difference was nonsignificant. The number of females responded about recoding notes about their previous and oncoming BSE was significantly higher in Group I compared to Group II, (Table 3).

There was non-significant difference between both groups as regards the method of performing the BSE, however, the applicability of circular motion was significantly higher in Group I compared to Group II and the use of either palm of hand of fingertips was significantly higher in Group I compared to Group II. The frequency of females doing BSE weekly and/or monthly was also significantly higher in Group Icompared to Group II, (Table 4). The feedback action to notice breast changes during BSE showed nonsignificant difference between both groups despite the increased frequency of asking a doctor or searching the net was higher among group I subjects, (Table 5, Fig. 2)

All females accustomed to perform BSE in group II had computer alone or in addition to internet facility, irrespective of being rural or urban inhabitant, while in group I about $35.7 \%$ of females had no computer facility with significantly higher frequency of having computer facility in group II. For both groups, irrespective of rural or urban inhabitance, the majority of females had their information through radio and TV, books and magazines are highly used by rural females of group I but by urban females of group II, other sources are nearly similar between both groups with significant difference of the frequency of source of information among groups according to location of inhabitance. Rural inhabitants performing BSE was significantly higher in both groups compared to urban inhabitants; however, timing of performing examination showed non-significant difference between both groups categorized according to site of inhabitance (Table 6).

There was significant difference between both groups categorized according to residence as regards knowledge about the methods of BSE, this was reflected as the significantly higher frequency of those interested in the breast appearance more than the presence of breast mass in rural women 
compared to urban women. This interest was documented by the method used for practicing BSE as manifested by significantly higher frequency of examination while standing in front of mirror (Table 7).

Educated mothers significantly affected their girls knowledge and application of facilities for gaining information as manifested by the significant difference of the availability of communication facilities and scientific sources of information as books and magazines and this was reflected on the frequency of practicing BSE which was significantly higher in both groups included educated mothers. Moreover, maternal education allowed significantly higher frequency of proper choice of timing of BSE; after menstruation in comparison to those whose mothers were illiterate, (Table 8). Mothers' education significantly affected the choice of method for practicing BSE with illiterate mothers taught their daughters to mainly use the observation and only to look for the mass, while educated mothers taught their daughters to observe the external shape, color and size of the breast and to also look for mass. Also, educated mothers taught their daughters to examine the whole breast with either fingertips or palm of hand and not to forget the axilla. Moreover, educated mothers advised their daughters to examine the breast while standing especially in front of the mirror so as to allow perfect observation and/or while lying down so as to allow proper touch. However, illiterate mothers advised their daughter to do BSE mostly while taking shower, (Table 9). 
Table (1): Distribution of the students according to socio-demographic characteristics among both groups

\begin{tabular}{|c|c|c|c|c|}
\hline & & $\begin{array}{l}\text { Specific } \\
\text { education } \\
(\text { no }=200)\end{array}$ & $\begin{array}{l}\text { Archaeology } \\
(\text { no=300) }\end{array}$ & $P$ value \\
\hline \multirow[t]{3}{*}{ Age (years) } & $17-20$ years & $155(77.5 \%)$ & $202(67.3 \%)$ & \multirow{3}{*}{$=0.009$} \\
\hline & $>20$ years & $45(22.5 \%)$ & $98(32.7 \%)$ & \\
\hline & Mean & $19.52 \pm 2.41$ & $19.75 \pm 2.74$ & \\
\hline \multirow{2}{*}{$\begin{array}{l}\text { Mother } \\
\text { education }\end{array}$} & Educated & $141(70.5 \%)$ & $186(62 \%)$ & \multirow{2}{*}{$=0.03$} \\
\hline & Illetrate & $59(29.5 \%)$ & $114(38 \%)$ & \\
\hline \multirow{2}{*}{$\begin{array}{l}\text { Mother } \\
\text { education }\end{array}$} & Educated & $167(83.5 \%)$ & $225(62 \%)$ & \multirow{2}{*}{$=0.015$} \\
\hline & Illetrate & $33(16.5 \%)$ & $75(38 \%)$ & \\
\hline \multirow[t]{2}{*}{ Residence } & Rural & $168(84 \%)$ & $155(51.7 \%)$ & \multirow{2}{*}{$<0.001$} \\
\hline & Urban & $32(16 \%)$ & $145(48.3 \%)$ & \\
\hline \multirow[t]{2}{*}{ Mother's job } & Housewives & $168(84 \%)$ & $233(77.7 \%)$ & \multirow{2}{*}{$>0.05$} \\
\hline & Employee & $32(16 \%)$ & $67(22.3 \%)$ & \\
\hline
\end{tabular}

Data are presented as mean \pm SD \& number; percentages are in parenthesis

Table (2): Distribution of the students according to knowledge about BSE

\begin{tabular}{|c|c|c|c|c|}
\hline & & $\begin{array}{c}\text { Specific } \\
\text { education } \\
(\mathrm{no}=200)\end{array}$ & $\begin{array}{l}\text { Archaeology } \\
(\text { no=300) }\end{array}$ & P value \\
\hline \multirow{2}{*}{$\begin{array}{l}\text { Knowledge } \\
\text { about BSE }\end{array}$} & Yes & $70(35 \%)$ & $65(21.7 \%)$ & \multirow{2}{*}{$=0.001$} \\
\hline & No & $130(65 \%)$ & $235(78.3 \%)$ & \\
\hline \multirow{7}{*}{$\begin{array}{l}\text { Source of } \\
\text { knowledge }\end{array}$} & Mother & $5(7.1 \%)$ & $8(12.3 \%)$ & \multirow{7}{*}{$=0.0163$} \\
\hline & $\begin{array}{ll}\text { Books and } \\
\text { magazines }\end{array}$ & $13(18.6 \%)$ & $8(12.3 \%)$ & \\
\hline & Radio \& TV & $23(32.9 \%)$ & $26(40 \%)$ & \\
\hline & Friends & $10(14.3 \%)$ & $3(4.6 \%)$ & \\
\hline & Family & $3(4.3 \%)$ & $5(7.7 \%)$ & \\
\hline & Computer \& Net & $11(15.7 \%)$ & $5(7.7 \%)$ & \\
\hline & Other & $5(7.1 \%)$ & $10(15.4 \%)$ & \\
\hline \multirow[t]{3}{*}{ Facilities } & Computer & $31(44.3 \%)$ & $26(40 \%)$ & \multirow{3}{*}{$>0.05$} \\
\hline & Computer \& Net & $17(24.3 \%)$ & $18(27.7 \%)$ & \\
\hline & $\mathrm{No}$ & $22(31.4 \%)$ & $21(32.3 \%)$ & \\
\hline \multirow{2}{*}{$\begin{array}{l}\text { Do you make } \\
\text { BSE }\end{array}$} & Yes & $35(50 \%)$ & $28(43.1 \%)$ & \multirow{2}{*}{$>0.05$} \\
\hline & No & $35(50 \%)$ & $37(56.9 \%)$ & \\
\hline \multirow{3}{*}{$\begin{array}{l}\text { How many } \\
\text { times you do } \\
\text { BSE }\end{array}$} & Every month & $10(28.6 \%)$ & $20(30.8 \%)$ & \multirow{3}{*}{$>0.05$} \\
\hline & Every 6 months & $14(40 \%)$ & $31(47.7 \%)$ & \\
\hline & Every year & $11(31.4 \%)$ & $14(21.5 \%)$ & \\
\hline \multirow{3}{*}{$\begin{array}{l}\text { What is the } \\
\text { exact date for } \\
\text { doing BSE }\end{array}$} & $\begin{array}{l}\text { Befroe } \\
\text { menstruation }\end{array}$ & $11(31.4 \%)$ & $7(25 \%)$ & \multirow{3}{*}{$>0.05$} \\
\hline & After menstruation & $11(31.4 \%)$ & $10(35.7 \%)$ & \\
\hline & No definite date & $13(37.2 \%)$ & $11(39.3 \%)$ & \\
\hline
\end{tabular}


Data are presented as number $\&$ percentages are in parenthesis

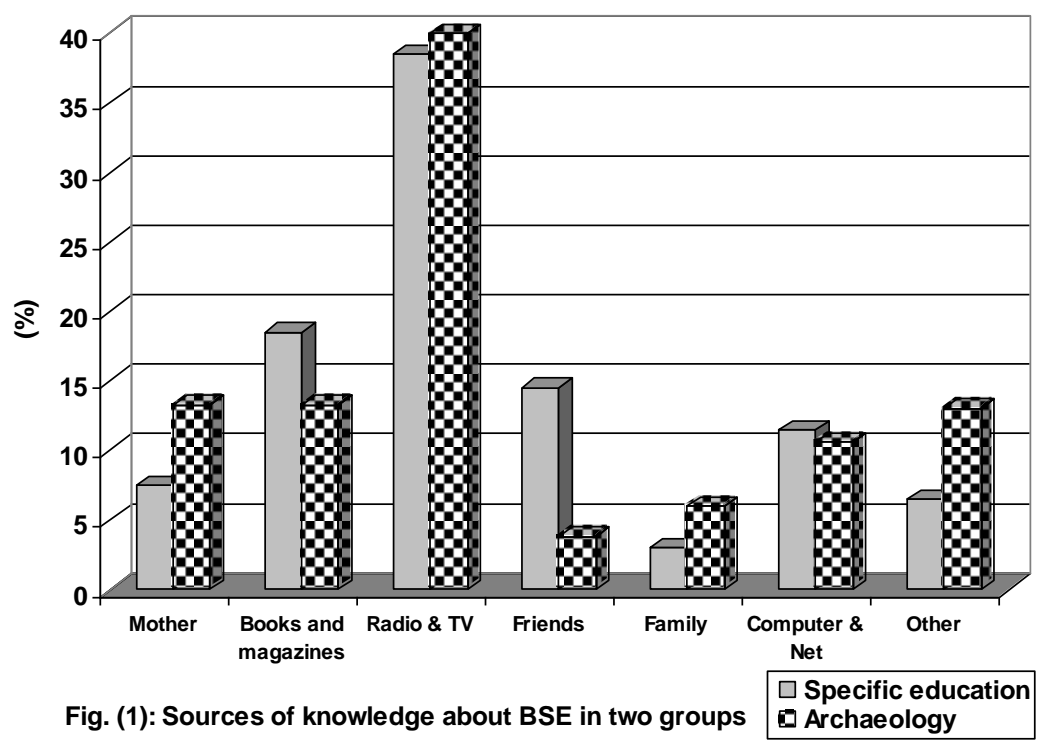

Table (3): Distribution of the students according to methods of BSE

\begin{tabular}{|c|c|c|c|c|}
\hline & & $\begin{array}{l}\text { Specific } \\
\text { education } \\
(\text { no }=70)\end{array}$ & $\begin{array}{l}\text { Archaeology } \\
\quad(\text { no }=65)\end{array}$ & $\mathrm{P}$ \\
\hline \multirow{5}{*}{$\begin{array}{l}\text { By } \\
\text { observation } \\
\text { and look }\end{array}$} & External shape & $19(27.1 \%)$ & $10(15.4 \%)$ & \multirow{5}{*}{$>0.05$} \\
\hline & Size of breast & $11(15.7 \%)$ & $10(15.4 \%)$ & \\
\hline & Color of breast & $5(7.1 \%)$ & $6(9.2 \%)$ & \\
\hline & abnormal mass & $20(28.6 \%)$ & $13(20 \%)$ & \\
\hline & All previous & $15(32.9 \%)$ & $26(40 \%)$ & \\
\hline \multirow{6}{*}{$\begin{array}{l}\text { By Touch \& } \\
\text { Pressure }\end{array}$} & Bellow collarbone & $5(7.1 \%)$ & $10(15.4 \%)$ & \multirow{6}{*}{$>0.05$} \\
\hline & $\begin{array}{l}\text { Touch breast by the three } \\
\text { middle fingers }\end{array}$ & $10(14.3 \%)$ & $15(23.1 \%)$ & \\
\hline & $\begin{array}{l}\text { Touch with circular } \\
\text { motion }\end{array}$ & $15(21.4 \%)$ & $15(23.1 \%)$ & \\
\hline & $\begin{array}{l}\text { Touch \& press all parts } \\
\text { of breast }\end{array}$ & $10(14.3 \%)$ & $5(7.7 \%)$ & \\
\hline & Exam lymph mode & $10(14.3 \%)$ & $5(7.7 \%)$ & \\
\hline & All previous & $20(28.6 \%)$ & $15(23.1 \%)$ & \\
\hline \multirow[t]{3}{*}{ By note } & Record notes & $10(14.3 \%)$ & $20(30.8 \%)$ & \multirow{3}{*}{$=0.01$} \\
\hline & $\begin{array}{l}\text { Determine the next time } \\
\text { of exam }\end{array}$ & $10(14.3 \%)$ & $15(23.1 \%)$ & \\
\hline & All previous & $50(71.4 \%)$ & $30(46.1 \%)$ & \\
\hline
\end{tabular}

Data are presented as number $\&$ percentages are in parenthesis 
Table (4): Distribution of students according to knowledge about methods preferring when doing exam in both group

\begin{tabular}{|c|c|c|c|c|}
\hline & & $\begin{array}{c}\text { Specific } \\
\text { education } \\
(\text { no }=70)\end{array}$ & $\begin{array}{l}\text { Archaeology } \\
\quad(\text { no }=65)\end{array}$ & $\mathrm{p}$ \\
\hline \multirow{4}{*}{$\begin{array}{l}\text { Methods } \\
\text { you prefer } \\
\text { during BSE }\end{array}$} & $\begin{array}{l}\text { While standing in front to } \\
\text { a mirror }\end{array}$ & $20(28.6 \%)$ & $15(23.1 \%)$ & \multirow{4}{*}{$>0.05$} \\
\hline & While lying down & $15(21.4 \%)$ & $20(30.7 \%)$ & \\
\hline & While taking a shower & $20(28.6 \%)$ & $15(23.1 \%)$ & \\
\hline & All previous & $15(21.4 \%)$ & $15(23.1 \%)$ & \\
\hline \multirow{6}{*}{$\begin{array}{l}\text { Mode of } \\
\text { BSE }\end{array}$} & Circular motion & $30(42.9 \%)$ & $10(15.4 \%)$ & \multirow{6}{*}{$=0.002$} \\
\hline & From enter to outer & $5(7.1 \%)$ & $10(15.4 \%)$ & \\
\hline & With clockwise & $20(28.6 \%)$ & $20(30.8 \%)$ & \\
\hline & From upper to lower & $10(14.3 \%)$ & $10(15.4 \%)$ & \\
\hline & From side to side & $5(7.1 \%)$ & $15(23.1 \%)$ & \\
\hline & Others & 0 & 0 & \\
\hline \multirow{4}{*}{$\begin{array}{l}\text { Part of hand } \\
\text { used for } \\
\text { BSE }\end{array}$} & The palm of the hand & $20(28.6 \%)$ & $20(30.8 \%)$ & \multirow{4}{*}{$<0.001$} \\
\hline & Fingertips & $35(50 \%)$ & $15(23.1 \%)$ & \\
\hline & All hands & $15(21.4 \%)$ & $10(15.4 \%)$ & \\
\hline & Others & 0 & $20(30.8 \%)$ & \\
\hline \multirow{4}{*}{$\begin{array}{l}\text { Exact time } \\
\text { for doing } \\
\text { BSE }\end{array}$} & Monthly & $30(42.9 \%)$ & $25(38.5 \%)$ & \multirow{4}{*}{$=0.03$} \\
\hline & Weekly & $15(21.4 \%)$ & $10(15.4 \%)$ & \\
\hline & $\begin{array}{l}7-10 \text { days from } 1^{\text {st }} \text { day of } \\
\text { menses }\end{array}$ & $20(28.6 \%)$ & $15(23.1 \%)$ & \\
\hline & $\begin{array}{l}\text { The same day every } \\
\text { month, irrespective of } \\
\text { menses }\end{array}$ & $5(7.1 \%)$ & $10(15.4 \%)$ & \\
\hline
\end{tabular}

Data are presented as number $\&$ percentages are in parenthesis

Table (5): Distribution of students according to action taken on finding any changes in their breast

\begin{tabular}{|l|c|c|c|}
\hline & Specific education (no=70) & $\begin{array}{c}\text { Archaeology } \\
(\text { no=65) }\end{array}$ & $\mathrm{p}$ \\
\hline Tell mother & $20(28.6 \%)$ & $15(23.1 \%)$ & \\
\hline Go to doctor & $20(28.6 \%)$ & $15(23.1 \%)$ & \multirow{2}{*}{$>0.05$} \\
\cline { 1 - 3 } Search in books \& net & $15(21.4 \%)$ & $10(15.4 \%)$ & $5(7.7 \%)$ \\
\cline { 1 - 3 } Go to friends & $5(7.1 \%)$ & $20(30.8 \%)$ & \\
\hline Others & $10(14.2 \%)$ & \\
\hline
\end{tabular}

Data are presented as number \& percentages are in parenthesis 


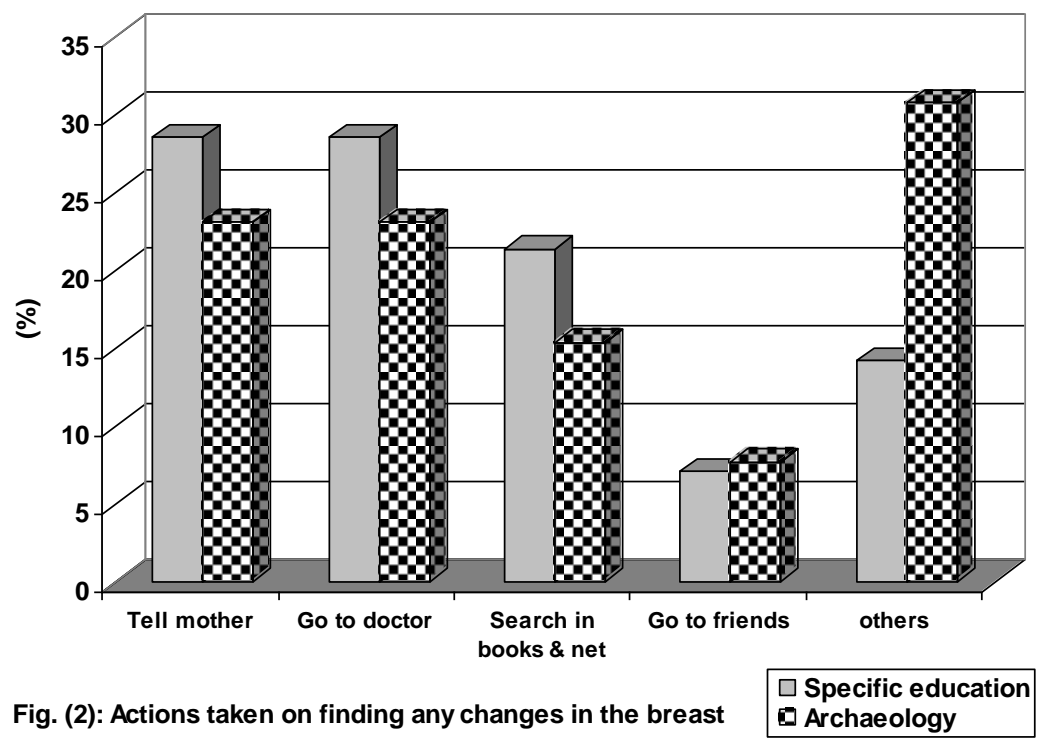

Table (6): The relation between residence $\&$ knowledge about BSE in both groups.

\begin{tabular}{|c|c|c|c|c|c|c|}
\hline & \multicolumn{2}{|c|}{ Specific education } & \multicolumn{2}{|c|}{ Archaeology } & \multirow[b]{2}{*}{$\mathrm{P}$} \\
\hline & & $\begin{array}{c}\text { Rural } \\
(\mathrm{n}=46)\end{array}$ & $\begin{array}{r}\text { Urban } \\
(\mathrm{n}=24)\end{array}$ & $\begin{array}{c}\text { Rural } \\
(\mathrm{n}=23)\end{array}$ & $\begin{array}{l}\text { Urban } \\
(\mathrm{n}=42)\end{array}$ & \\
\hline \multirow[t]{3}{*}{ Facilities } & Computer & $\begin{array}{c}30 \\
(65.2 \%)\end{array}$ & 0 & $\begin{array}{c}13 \\
(65.5 \%)\end{array}$ & $21(50 \%)$ & \multirow{3}{*}{$<0.001$} \\
\hline & Computer \& Net & $\begin{array}{c}5 \\
(10.9 \%)\end{array}$ & $\begin{array}{c}10 \\
(41.7 \%)\end{array}$ & $\begin{array}{c}10 \\
(44.5 \%)\end{array}$ & $21(50 \%)$ & \\
\hline & No & $\begin{array}{c}11 \\
(23.9 \%)\end{array}$ & $\begin{array}{c}14 \\
(58.3 \%)\end{array}$ & 0 & 0 & \\
\hline \multirow[t]{7}{*}{$\begin{array}{l}\text { Source of } \\
\text { infromation }\end{array}$} & Mother & $\begin{array}{c}5 \\
(10.9 \%)\end{array}$ & - & $3(13 \%)$ & $\begin{array}{c}6 \\
(14.3 \%)\end{array}$ & \multirow[t]{7}{*}{$=0.003$} \\
\hline & $\begin{array}{ll}\text { Books } & \text { and } \\
\text { magazines }\end{array}$ & $\begin{array}{c}13 \\
(28.3 \%)\end{array}$ & - & - & $\begin{array}{c}9 \\
(21.4 \%)\end{array}$ & \\
\hline & Radio \& TV & $6(13 \%)$ & $\begin{array}{c}21 \\
(87.5 \%)\end{array}$ & $\begin{array}{c}10 \\
(44.5 \%)\end{array}$ & $\begin{array}{c}16 \\
(38.1 \%)\end{array}$ & \\
\hline & Friends & $\begin{array}{c}10 \\
(21.7 \%)\end{array}$ & - & - & $2(4.8 \%)$ & \\
\hline & Family & $1(2.2 \%)$ & - & $\begin{array}{c}4 \\
(17.4 \%)\end{array}$ & - & \\
\hline & Computer \& Net & $\begin{array}{c}7 \\
(15.2 \%)\end{array}$ & $1(4.2 \%)$ & $3(13 \%)$ & $4(9.5 \%)$ & \\
\hline & Other & $4(8.7 \%)$ & $2(8.3 \%)$ & $3(13 \%)$ & $\begin{array}{c}5 \\
(11.9 \%)\end{array}$ & \\
\hline \multirow[t]{2}{*}{ Doing BSE } & Yes & $\begin{array}{c}25 \\
(54.3 \%)\end{array}$ & $\begin{array}{c}10 \\
(41.7 \%)\end{array}$ & $\begin{array}{c}15 \\
(65.2 \%)\end{array}$ & $13(31 \%)$ & \multirow{2}{*}{$=0.008$} \\
\hline & No & $\begin{array}{c}21 \\
(45.7 \%)\end{array}$ & $\begin{array}{c}14 \\
(58.3 \%)\end{array}$ & $\begin{array}{c}8 \\
(34.2 \%)\end{array}$ & $29(69 \%)$ & \\
\hline \multirow[t]{3}{*}{$\begin{array}{l}\text { Time of } \\
\text { BSE }\end{array}$} & $\begin{array}{l}\text { Befroe } \\
\text { menstruation }\end{array}$ & $\begin{array}{c}15 \\
(32.6 \%) \\
\end{array}$ & $\begin{array}{c}5 \\
(20.8 \%) \\
\end{array}$ & $\begin{array}{c}10 \\
(66.7 \%) \\
\end{array}$ & $\begin{array}{c}12 \\
(92.3 \%) \\
\end{array}$ & \multirow{3}{*}{$>0.05$} \\
\hline & $\begin{array}{l}\text { After } \\
\text { menstruation }\end{array}$ & $\begin{array}{c}14 \\
(30.4 \%)\end{array}$ & $\begin{array}{c}16 \\
(66.7 \%)\end{array}$ & $3(20 \%)$ & $1(7.7 \%)$ & \\
\hline & No definite date & $17(37 \%)$ & $\begin{array}{c}3 \\
(12.5 \%)\end{array}$ & $\begin{array}{c}2 \\
(13.3 \%)\end{array}$ & 0 & \\
\hline
\end{tabular}

Data are presented as number $\&$ percentages are in parenthesis 
Table (7): Relation between residence \& knowledge about methods of BSE in both groups

\begin{tabular}{|c|c|c|c|c|c|c|}
\hline & \multicolumn{2}{|c|}{ Specific education } & \multicolumn{2}{|c|}{ Archaeology } & \multirow[t]{2}{*}{$\mathrm{P}$} \\
\hline & & $\begin{array}{l}\text { Rural } \\
(n=46)\end{array}$ & $\begin{array}{l}\text { Urban } \\
(\mathrm{n}=24)\end{array}$ & $\begin{array}{l}\text { Rural } \\
(n=23)\end{array}$ & $\begin{array}{l}\text { Urban } \\
(\mathrm{n}=42)\end{array}$ & \\
\hline \multirow{5}{*}{$\begin{array}{l}\text { By } \\
\text { observation } \\
\text { and look }\end{array}$} & External shape & $17(37 \%)$ & $2(8.3 \%)$ & $\begin{array}{c}7 \\
(30.8 \%)\end{array}$ & $3(7.1 \%)$ & \multirow{5}{*}{$<0.001$} \\
\hline & Size of breast & $\begin{array}{c}11 \\
(23.9 \%)\end{array}$ & 0 & $\begin{array}{c}7 \\
(30.8 \%)\end{array}$ & $3(7.1 \%)$ & \\
\hline & Color of breast & $3(6.5 \%)$ & $2(8.3 \%)$ & $\begin{array}{c}4 \\
(17.4 \%)\end{array}$ & $2(4.8 \%)$ & \\
\hline & Abnormal mass & $\begin{array}{c}5 \\
(10.9 \%)\end{array}$ & $\begin{array}{c}15 \\
(62.5 \%)\end{array}$ & $2(8.7 \%)$ & $\begin{array}{c}11 \\
(26.2 \%)\end{array}$ & \\
\hline & All previous & $\begin{array}{c}10 \\
(21.7 \%)\end{array}$ & $\begin{array}{c}5 \\
(20.8 \%)\end{array}$ & $3(13 \%)$ & $\begin{array}{c}23 \\
(54.8 \%)\end{array}$ & \\
\hline \multirow[t]{6}{*}{$\begin{array}{l}\text { By Touch } \\
\text { \&Pressure }\end{array}$} & $\begin{array}{l}\text { Bellow } \\
\text { collarbone }\end{array}$ & $\begin{array}{c}5 \\
(10.9 \%)\end{array}$ & 0 & $\begin{array}{c}8 \\
(34.8 \%)\end{array}$ & $2(4.8 \%)$ & \multirow{6}{*}{$<0.001$} \\
\hline & $\begin{array}{l}\text { Touch breast by } \\
\text { the three } \\
\text { fingertips }\end{array}$ & $\begin{array}{c}10 \\
(21.7 \%)\end{array}$ & 0 & $\begin{array}{c}8 \\
(34.8 \%)\end{array}$ & $7(16.7)$ & \\
\hline & Circular motion & $\begin{array}{c}12 \\
(26.1 \%)\end{array}$ & $\begin{array}{c}3 \\
(12.5 \%)\end{array}$ & $\begin{array}{c}4 \\
(17.4 \%)\end{array}$ & $11(26.2)$ & \\
\hline & $\begin{array}{l}\text { Touch \& press } \\
\text { all parts of } \\
\text { breast }\end{array}$ & $6(13 \%)$ & $\begin{array}{c}4 \\
(16.7 \%)\end{array}$ & 0 & $\begin{array}{c}0 \\
(11.9 \%)\end{array}$ & \\
\hline & $\begin{array}{l}\text { Exam lymph } \\
\text { mode }\end{array}$ & $\begin{array}{c}7 \\
(15.2 \%)\end{array}$ & $\begin{array}{c}3 \\
(12.5 \%)\end{array}$ & 0 & $\begin{array}{c}0 \\
(11.9 \%)\end{array}$ & \\
\hline & All previous & $6(13 \%)$ & $\begin{array}{c}14 \\
(58.3 \%)\end{array}$ & $3(13 \%)$ & $\begin{array}{c}12 \\
(28.6 \%)\end{array}$ & \\
\hline \multirow[t]{3}{*}{ Notes } & Record notes & $\begin{array}{c}10 \\
(21.7 \%) \\
\end{array}$ & 0 & $\begin{array}{c}13 \\
(56.5 \%)\end{array}$ & $\begin{array}{c}7 \\
(16.7 \%)\end{array}$ & \multirow{3}{*}{$<0.001$} \\
\hline & $\begin{array}{l}\text { Determine next } \\
\text { time }\end{array}$ & $\begin{array}{c}10 \\
(21.7 \%)\end{array}$ & 0 & $3(13 \%)$ & $\begin{array}{c}12 \\
(28.6 \%)\end{array}$ & \\
\hline & All previous & $\begin{array}{c}26 \\
(56.5 \%)\end{array}$ & $\begin{array}{c}24 \\
(100 \%)\end{array}$ & $\begin{array}{c}7 \\
(30.4 \%)\end{array}$ & $\begin{array}{c}23 \\
(54.8 \%)\end{array}$ & \\
\hline \multirow{4}{*}{$\begin{array}{l}\text { Methods } \\
\text { you prefer } \\
\text { during } \\
\text { BSE }\end{array}$} & $\begin{array}{l}\text { While standing } \\
\text { in front to a } \\
\text { mirror }\end{array}$ & $\begin{array}{c}20 \\
(43.5 \%)\end{array}$ & 0 & $\begin{array}{c}11 \\
(47.8 \%)\end{array}$ & $4(9.5 \%)$ & \multirow{4}{*}{$<0.001$} \\
\hline & $\begin{array}{l}\text { While lying } \\
\text { down }\end{array}$ & $\begin{array}{c}12 \\
(26.1 \%)\end{array}$ & $\begin{array}{c}3 \\
(12.5 \%)\end{array}$ & $2(8.7 \%)$ & $\begin{array}{c}18 \\
(42.9 \%)\end{array}$ & \\
\hline & $\begin{array}{l}\text { While taking a } \\
\text { shower }\end{array}$ & $\begin{array}{c}8 \\
(17.4 \%)\end{array}$ & $12(50 \%)$ & $\begin{array}{c}8 \\
(34.8 \%)\end{array}$ & $\begin{array}{c}7 \\
(16.7 \%)\end{array}$ & \\
\hline & All previous & $6(13 \%)$ & $\begin{array}{c}9 \\
(37.5 \%)\end{array}$ & $2(8.7 \%)$ & $13(31 \%)$ & \\
\hline
\end{tabular}

Data are presented as number $\&$ percentages are in parenthesis 
Table (8): Relation between mother education and knowledge about BSE among both groups

\begin{tabular}{|c|c|c|c|c|c|c|}
\hline & \multicolumn{2}{|c|}{ Specific education } & \multicolumn{2}{|c|}{ Archaeology } & \multirow[t]{2}{*}{$\mathrm{P}$} \\
\hline & & $\begin{array}{c}\text { Educated } \\
(n=49)\end{array}$ & $\begin{array}{c}\text { Illiterate } \\
(\mathrm{n}=21)\end{array}$ & $\begin{array}{c}\text { Educated } \\
(\mathrm{n}=47)\end{array}$ & $\begin{array}{c}\text { Illiterate } \\
(\mathrm{n}=18)\end{array}$ & \\
\hline \multirow[t]{3}{*}{ Facilities } & Computer & $\begin{array}{c}30 \\
(61.2 \%)\end{array}$ & 0 & $\begin{array}{c}21 \\
(44.7 \%)\end{array}$ & 0 & \multirow{3}{*}{$<0.001$} \\
\hline & $\begin{array}{l}\text { Computer \& } \\
\text { Net }\end{array}$ & $\begin{array}{c}5 \\
(10.2 \%)\end{array}$ & $\begin{array}{c}10 \\
(47.6 \%)\end{array}$ & $16(34 \%)$ & $\begin{array}{c}10 \\
(55.6 \%)\end{array}$ & \\
\hline & No & $\begin{array}{c}14 \\
(28.6 \%)\end{array}$ & $\begin{array}{c}11 \\
(52.4 \%)\end{array}$ & $\begin{array}{c}10 \\
(21.3 \%)\end{array}$ & $\begin{array}{c}8 \\
(44.4 \%)\end{array}$ & \\
\hline \multirow[t]{7}{*}{$\begin{array}{l}\text { Source of } \\
\text { infromation }\end{array}$} & Mother & $\begin{array}{c}5 \\
(10.2 \%)\end{array}$ & 0 & $\begin{array}{c}9 \\
(19.1 \%)\end{array}$ & 0 & \multirow{7}{*}{$=0.003$} \\
\hline & $\begin{array}{ll}\text { Books } & \text { and } \\
\text { magazines } & \end{array}$ & $\begin{array}{c}13 \\
(26.5 \%)\end{array}$ & 0 & $8(17 \%)$ & $1(5.6)$ & \\
\hline & Radio \& TV & $\begin{array}{c}6 \\
(12.2 \%)\end{array}$ & $\begin{array}{c}21 \\
(100 \%)\end{array}$ & $16(34 \%)$ & $\begin{array}{c}10 \\
(55.6 \%) \\
\end{array}$ & \\
\hline & Friends & $\begin{array}{c}10 \\
(22.4 \%)\end{array}$ & 0 & $1(2.1 \%)$ & $1(5.6)$ & \\
\hline & Family & $1(2 \%)$ & 0 & $4(8.5 \%)$ & 0 & \\
\hline & $\begin{array}{l}\text { Computer } \\
\text { Net }\end{array}$ & $\begin{array}{c}8 \\
(16.3 \%) \\
\end{array}$ & 0 & $\begin{array}{c}5 \\
(10.6 \%) \\
\end{array}$ & $\begin{array}{c}2 \\
(11.1 \%)\end{array}$ & \\
\hline & Other & $\begin{array}{c}6 \\
(12.2 \%)\end{array}$ & 0 & $4(8.5 \%)$ & $\begin{array}{c}4 \\
(22.2 \%)\end{array}$ & \\
\hline \multirow[t]{2}{*}{ Doing BSE } & Yes & $\begin{array}{c}28 \\
(57.1 \%)\end{array}$ & $\begin{array}{c}7 \\
(33.3 \%)\end{array}$ & $\begin{array}{c}25 \\
(53.2 \%)\end{array}$ & $\begin{array}{c}3 \\
(16.7 \%)\end{array}$ & \multirow{2}{*}{$=0.003$} \\
\hline & No & $\begin{array}{c}21 \\
(42.9 \%)\end{array}$ & $\begin{array}{c}14 \\
(28.6 \%)\end{array}$ & $\begin{array}{c}22 \\
(46.8 \%)\end{array}$ & $\begin{array}{c}7 \\
(83.3 \%)\end{array}$ & \\
\hline \multirow[t]{3}{*}{$\begin{array}{l}\text { Time of } \\
\text { BSE }\end{array}$} & $\begin{array}{l}\text { Befroe } \\
\text { menstruation }\end{array}$ & $\begin{array}{c}18 \\
(36.7 \%)\end{array}$ & 0 & $\begin{array}{c}15 \\
(31.9 \%)\end{array}$ & 0 & \multirow{3}{*}{$<0.001$} \\
\hline & $\begin{array}{l}\text { After } \\
\text { menstruation }\end{array}$ & $\begin{array}{c}13 \\
(26.5 \%)\end{array}$ & $\begin{array}{c}24 \\
(100 \%)\end{array}$ & $\begin{array}{c}21 \\
(44.7 \%)\end{array}$ & $\begin{array}{c}8 \\
(44.4 \%)\end{array}$ & \\
\hline & No definite date & $\begin{array}{c}18 \\
(36.7 \%)\end{array}$ & 0 & $\begin{array}{c}11 \\
(23.4 \%)\end{array}$ & $\begin{array}{c}10 \\
(55.6 \%)\end{array}$ & \\
\hline
\end{tabular}

Data are presented as number \& percentages are in parenthesis 
Table (9): The Relation between methods of BSE and education of mother among both groups.

\begin{tabular}{|c|c|c|c|c|c|c|}
\hline & \multicolumn{2}{|c|}{ Specific education } & \multicolumn{2}{|c|}{ Archaeology } & \multirow[t]{2}{*}{$\mathrm{P}$} \\
\hline & & $\begin{array}{l}\text { Educated } \\
(\mathrm{n}=49)\end{array}$ & $\begin{array}{c}\text { Illiterate } \\
(n=21)\end{array}$ & $\begin{array}{l}\text { Educated } \\
(n=47)\end{array}$ & $\begin{array}{c}\text { Illiterate } \\
(n=18)\end{array}$ & \\
\hline \multirow{5}{*}{$\begin{array}{l}\text { By } \\
\text { observation } \\
\text { and look }\end{array}$} & External shape & $\begin{array}{c}19 \\
(38.8 \%)\end{array}$ & 0 & $\begin{array}{c}10 \\
(21.3 \%)\end{array}$ & 0 & \multirow{5}{*}{$<0.001$} \\
\hline & Size of breast & $\begin{array}{c}11 \\
(22.4 \%) \\
\end{array}$ & 0 & $\begin{array}{c}10 \\
(21.3 \%) \\
\end{array}$ & 0 & \\
\hline & Color of breast & $4(8.2 \%)$ & $1(4.8 \%)$ & $\begin{array}{c}6 \\
(12.8 \%)\end{array}$ & 0 & \\
\hline & Abnormal mass & $\begin{array}{c}6 \\
(12.2 \%)\end{array}$ & $\begin{array}{c}14 \\
(66.7 \%)\end{array}$ & $\begin{array}{c}11 \\
(23.4 \%)\end{array}$ & $\begin{array}{c}2 \\
(11.1 \%)\end{array}$ & \\
\hline & All previous & $\begin{array}{c}9 \\
(18.4 \%)\end{array}$ & $\begin{array}{c}6 \\
(28.6 \%)\end{array}$ & $\begin{array}{c}10 \\
(21.3 \%)\end{array}$ & $\begin{array}{c}16 \\
(88.9 \%)\end{array}$ & \\
\hline \multirow[t]{6}{*}{$\begin{array}{l}\text { By Touch } \\
\text { \&Pressure }\end{array}$} & $\begin{array}{l}\text { Bellow } \\
\text { collarbone }\end{array}$ & $\begin{array}{c}5 \\
(10.2 \%)\end{array}$ & 0 & $\begin{array}{c}10 \\
(21.3 \%)\end{array}$ & 0 & \multirow{6}{*}{$<0.001$} \\
\hline & $\begin{array}{l}\text { Touch breast by } \\
\text { the three } \\
\text { fingertips }\end{array}$ & $\begin{array}{c}10 \\
(20.4 \%)\end{array}$ & 0 & $\begin{array}{c}15 \\
(31.9 \%)\end{array}$ & 0 & \\
\hline & Circular motion & $\begin{array}{c}12 \\
(24.5 \%)\end{array}$ & $\begin{array}{c}3 \\
(14.3 \%)\end{array}$ & $\begin{array}{c}9 \\
(19.1 \%)\end{array}$ & $\begin{array}{c}6 \\
(33.3 \%) \\
\end{array}$ & \\
\hline & $\begin{array}{l}\text { Touch \& press } \\
\text { all parts of } \\
\text { breast }\end{array}$ & $\begin{array}{c}9 \\
(18.4 \%)\end{array}$ & $1(4.8 \%)$ & $4(8.5 \%)$ & $1(5.6 \%)$ & \\
\hline & $\begin{array}{ll}\text { Exam lymph } \\
\text { mode }\end{array}$ & $\begin{array}{c}7 \\
(14.3 \%)\end{array}$ & $\begin{array}{c}3 \\
(14.3 \%)\end{array}$ & $2(4.3 \%)$ & $\begin{array}{c}3 \\
(16.7 \%)\end{array}$ & \\
\hline & All previous & $\begin{array}{c}6 \\
(12.2 \%)\end{array}$ & $\begin{array}{c}14 \\
(66.7 \%)\end{array}$ & $\begin{array}{c}7 \\
(14.9 \%)\end{array}$ & $\begin{array}{c}8 \\
(44.4 \%)\end{array}$ & \\
\hline \multirow[t]{3}{*}{ Notes } & Record notes & $\begin{array}{c}10 \\
(20.4 \%) \\
\end{array}$ & 0 & $\begin{array}{c}20 \\
(42.6 \%) \\
\end{array}$ & 0 & \multirow{3}{*}{$=0.002$} \\
\hline & $\begin{array}{l}\text { Determine next } \\
\text { time }\end{array}$ & $\begin{array}{c}10 \\
(20.4 \%)\end{array}$ & 0 & $\begin{array}{c}10 \\
(21.3 \%)\end{array}$ & $\begin{array}{c}5 \\
(27.8 \%)\end{array}$ & \\
\hline & All previous & $\begin{array}{c}29 \\
(59.2 \%)\end{array}$ & $\begin{array}{c}21 \\
(100 \%)\end{array}$ & $\begin{array}{c}17 \\
(36.2 \%)\end{array}$ & $\begin{array}{c}13 \\
(72.2 \%)\end{array}$ & \\
\hline \multirow{4}{*}{$\begin{array}{l}\text { Methods } \\
\text { you prefer } \\
\text { during } \\
\text { BSE }\end{array}$} & $\begin{array}{l}\text { In front to a } \\
\text { mirror }\end{array}$ & $\begin{array}{c}18 \\
(36.7 \%)\end{array}$ & $2(9.5 \%)$ & $\begin{array}{c}15 \\
(31.9 \%)\end{array}$ & 0 & \multirow{4}{*}{$=0.001$} \\
\hline & $\begin{array}{ll}\text { While lying } \\
\text { down }\end{array}$ & $\begin{array}{c}15 \\
(30.6 \%) \\
\end{array}$ & 0 & $8(17 \%)$ & $\begin{array}{c}12 \\
(66.7 \%) \\
\end{array}$ & \\
\hline & Taking a shower & $\begin{array}{c}10 \\
(20.4 \%)\end{array}$ & $\begin{array}{c}10 \\
(47.6 \%)\end{array}$ & $\begin{array}{c}13 \\
(27.7 \%)\end{array}$ & $\begin{array}{c}2 \\
(11.1 \%)\end{array}$ & \\
\hline & All previous & $\begin{array}{c}6 \\
(12.2 \%)\end{array}$ & $\begin{array}{c}9 \\
(42.9 \%)\end{array}$ & $\begin{array}{c}11 \\
(23.4 \%)\end{array}$ & $\begin{array}{c}4 \\
(22.2 \%)\end{array}$ & \\
\hline
\end{tabular}

Data are presented as number \& percentages are in parenthesis 


\section{Discussion}

The current study reported that about $65 \%$ of Specific Education Faculty sample (Group I) and $78.3 \%$ of Archaeology Faculty sample (Group II) do not have knowledge about BSE. This finding agrees with that previously reported in literature; Seif \& Aziz ${ }^{(9)}$ found that about $25 \%$ of a group of working women in Ain Shams University survey about effect of BSE training program on knowledge, attitude and practice heard about breast self examination. Heidari et al. ${ }^{(10)}$ reported that $21.6 \%$ of women residing in South East of Iran had good knowledge about BSE. Balogun (11) found $31.7 \%$ of female Traders in Ibadan, Oyo state, Nigeria were aware of BSE.

The present study also reflects that, less than one-fifth of female students (18.6\% \& $13.8 \%$ respectively) in both groups obtained their knowledge from books and magazine. This finding goes in hand with Seif \& Aziz (9) who showed that books represented $13.1 \%$ as source of knowledge about BSE. Also, Adenik et al. ${ }^{(12)}$, (2009) conducted a study of knowledge, practice of female health workers in a Nigerians urban city and found $31.1 \%$ obtain information from journals and Yan ${ }^{(13)}$ found mass media such as newspaper as source of information for about $37.1 \%$ of study population.

About $40 \%$ of study participants depended on Radio and TV for obtaining their knowledge about BSE; this figure supported that previously reported by Seif \& Aziz ${ }^{(9)}$ who found Radio and TV represent about 30\% of the sources of information about BSE, but was contradictory to Demirkian et al. ${ }^{(14)}$ who found that among nurses \& teachers in Turkey the most important information sources for both groups were books \& Magazines. On the other side, Yan ${ }^{(13)}$ mentioned that Radio and TV was the major information source of BSE for about $58.6 \%$ of study participants. These findings spot light on the fact that effort should be intensified in using these media to create BSE awareness and emphasize the importance of early detection as this appears to be better media to reach a wider audience.

As regards the effect of interpersonal communications as a source of knowledge, about $10 \%$ studied female students got their information from 
friends. The reported figure agreed Adenik et al. ${ }^{(12)}$ who found that in a Nigerian urban city, only $4.1 \%$ of study participants obtained information from their colleagues. However, the obtained figure was contradictory to the work of Seif \& Aziz ${ }^{(9)}$ who found that in Cairo, Egypt the main source of information about BSE was peer group (47.8\%), but this high figure could be attributed to the long time that working women spent with each other at the workplace, discussing different issues, which creates strong relation among each other.

In the present study, it is found that about $50 \%$ of female students who had knowledge about BSE do it with nonsignificant difference between study groups. The reported figure superceded that reported by Heidari et al. (10), Milaat $^{(15)}$ and Tanjasiri et al. ${ }^{(16)}$ and who reported figures of $23.3 \%, 12 \%$ and $37 \%$ for performing BSE among females had the knowledge about it, respectively.

Concerning the frequency of BSE, about $30 \%$ of study participants who do BSE conduct it monthly, a figure that superceded that reported by Alkhasawneh ${ }^{(17)}$ who found only 18 of
Jordanian women practice BSE on monthly basis, Pinar et al. ${ }^{(18)}$ who reported a figure of $10.2 \%$ among a group of women in a rural area in Western Turkey. About 50\% of females practicing BSE monthly do it after the end of menstrual period and about $30 \%$ of them do it before the period and the remaining had no definite date for examination.

Review of literature concerning timing of BSE was discrepant and this could be attribute to the extent of knowledge in relation to age, level of education and type of work of study participants; Milaat ${ }^{(15)}$ reported that only $14.4 \%$ of secondary-school female students had knowledge about time of BSE. Demirkian et al. ${ }^{(14)}$ reported that $69.3 \%$ and $46.7 \%$ of studied nurses and teachers, respectively, had a knowledge about proper timing of BSE and attributed this difference to the fact that nurses have correct knowledge about BSE procedures because of their profession.

As regards mode of practicing BSE about $30 \%$ of those do BSE prefer to do it while standing either in front of the mirror or during taking shower and mostly use their palm of hand for 
examination and continuously observe their breasts and look for masses. These data go in hand with Rosemary, et al., (2010) who conducted a study in Lagos among Nursing students, showed that about $50 \%$ of students prefer doing BSE in front a mirror and about $28 \%$ prefer BSE while taking a shower; however, higher percentage $(85.6 \%)$ use their palms during BSE, this could be attributed to the fact that this group consider proficient group because of their job or field of work.

The present study showed a highly significant difference between both study groups regarding to facilities and methods of observation in relation to residence and mother education. It could be concluded that knowledge about breast self examination is still underdeveloped and controlled by the residence, level of education of the mothers, the availability of knowledge providing facilities and even these knowledge needed to be corrected as regards how and when to practice BSE. Thus mass surveys for provision of knowledge among higher school and university student is mandatory to develop and correct their knowledge about BSE

\section{References}

1. World Health Organization: Global Strategy on Diet, physical Activity and Health, 2003. Available at: www.who.intlcancer.

2. Ferlay, Autier P, Bonio 1M: Estimates of the cancer incidence and mortality in Europe, 2006; 18(3):581.

3. Omar SH, Khaled R, Gaafar AR, Zekry S, Eissa1 and O. El-Khatib: Breast cancer in Egypt: a review of disease presentation and detection strategies, 1st ed Korba International: London, 2003; 9(3): 15-8.

4. Howard Fuchsia RN, MSN, Shannon Scott-Findlay RN, BN Article first published online. Breast self examination, 2006: 10: 66-70.

5. Saludeen AG, Akande TM, Musa OI: Knowledge and Attitudes to breast cancer and breast self examination among female undergraduates in a state in Nigeria. European J. Social Sci., 2009; (7):157-65.

6. American Cancer Society: Updated breast cancer screening guidelines released, 2006. Available at www.cancer.org.

7. National Cancer Institute: Summery of evidence, screening breast self 
examination, 2009. Available at www.cancer govlbreastlpage5.

8. National Cancer Institute: Breast and Prostate Cancer and Hormone-Related Gene Variants Cohort Consortium, 2009. Available at: www. epi.grants.cancer.gov.

9. Seif N, Aziz M: Effect of BSE training program on knowledge, Attitudes and practice of a Group of working women. Journal of the Egyptian Nat. Cancer Inst., 2000; 12(2):105-15.

10. Heidari Z, Mahmoudzadeh-Sagheb1 HR, Sakhavar N: breast cancer screening knowledge and practice among women in South East of Iran, Zehedan University of Medical sciences, 2003.

11. Balogun MO, Owoaje ET: Knowledge and practice of breast cancer screening among female traders in lbadan, Nigeria Department of Community Medicine, University College Hospital, Ibadan, Oyo State, Nigeria. Annals of Ibadan Postgraduate Medicine, 2003;. 32(2):99-102.

12. Adenik, Omuemu: knowledge, attitudes and practice of breast cancer screening among female health workers in a Nigerian urban city.,
2009; 4(9)\147-203. Available at www.biomedcentral.com.

13. Yan YY: Breast cancer: knowledge and perceptions of Chinese women in Hong Kong. Global J. Health Sci., 2009; (1): 97-105.

14. Demirkian F, Memis S, Turk G: How do nurses and teachers perform BSE: are they reliable sources of information?, 2004; (6): 234238.Available at: www.biomed central.

15. Milaat WA: knowledge of secondary school female students on breast cancer and BSE in Jeddah, Saudi Arabia. East Medite, 2000;(6):338-44.

16. Tanjasiri SP, Tanjasiri SP, SablanSantosl: Breast cancer screening among Chamorro women in southern California. J Women's Health, 2000; 10(5):479- 85 .

17. Alkhasawneh E: Barriers to and predictors of the practice of breast selfexamination in Jordanian Muslim women. Unpublished doctoral dissertation, State University of New York, Buffalo, 2002.

18. Pinar ,Erbay Dilek Özmen, Beyhan Öztürk, Gökçe Haspolat, Filiz Akyıldız, Sümeyra Çoban, and Gamze Çakıroğlu:The knowledge and attitudes 
19. of breast self-examination and mammography in a group of women in a rural area in western Turkey pub med central BMC Cancer, 2006; (6): 43.

20. Rosemary B, Bassey,Nicholas K: knowledge, Attitude and practice of Breast Self-Examination among Nursing students in Lagos University Teaching Hospital .Academic Journal of cancer Research, 2010; 3(2):11-15. 
